\title{
S26-01
}

\section{PATIENTS WITH FIRST EPISODE PSYCHOSIS TAKE MORE AND STRONGER CANNABIS THAN CONTROLS}

\section{Di Forti}

General Psychiatry, Institute of Psychiatry, Psychological Medicine, London, UK

Epidemiological studies have reported that the increased risk of developing psychosis in cannabis users is dose related. In addition, experimental research has shown that the active constituent of cannabis responsible for its psychotogenic effect is Delta-9Tetrahydrocannabinol (THC) (Murray et al, 2007). Recent evidence has suggested an increased in potency (\% TCH) in the cannabis seized in the UK (Potter et al, 2007). We predicted that first episode psychosis patients are more likely to use higher potency cannabis and more frequently than controls.

We collected information concerning socio-demographic, clinical characteristics and cannabis use (age at first use, frequency, length of use, type of cannabis used) from a sample of 191 first-episode psychosis patients and 120 matched healthy volunteers Cases were more likely to be regular users $(p=0.05)$, to be current users $(p=0.04)$ and to have smoked cannabis for longer $(p=0.01)$. Among cannabis users, $\mathbf{8 6 . 8 \%}$ of 1 st Episode Psychosis Patients preferentially used Skunk/Sinsemilla compared to $\mathbf{2 7 . 7 \%}$ of Controls. Only $\mathbf{1 3 . 2} \%$ of 1 st Episode psychosis Patients chose to use Resin/Hash compared to $\mathbf{7 6 . 3 \%}$ of controls $\left(\mathbf{O R} \mathbf{R}^{*} \mathbf{7 . 4} \mathbf{9 5 \%} \mathrm{Cl}\right.$ 3.4- 17.2, Adjusted for age, gender, ethnicity, level of Education and employment status). The concentration of TCH in these in South East London, ranges between 8.5 and $14 \%$ (Potter et al, 2007). Controls (47\%) were more likely to use Hash (Resin) whose average TCH concentration is $3.4 \%$ (Potter et al, 2007).

Patients with first episode psychosis have smoked higher potency cannabis, for longer and with greater frequency, than healthy controls. 